\title{
35.
}

\section{ON HOMOGENEOUS FUNCTIONS OF THE THIRD ORDER WITH THREE VARIABLES.}

[From the Cambridge and Dublin Mathematical Journal, vol. I. (1846), pp. 97-104.]

THE following problem corresponds to the geometrical question of determining the polar reciprocal of a plane curve of the third order: the solution of it is also important, with reference to the linear transformations of homogeneous functions of three variables of the third order; reasons for which it has appeared to me worth while to obtain the completely developed result.

Let

$3 U=a x^{3}+b y^{3}+c z^{3}+3 i y^{2} z+3 j z^{2} x+3 k x^{2} y+3 i_{1} y z^{2}+3 j_{1} z x^{2}+3 k_{1} x y^{2}+6 i x y z$

It is required to eliminate $x, y, z, \lambda$ from the equations

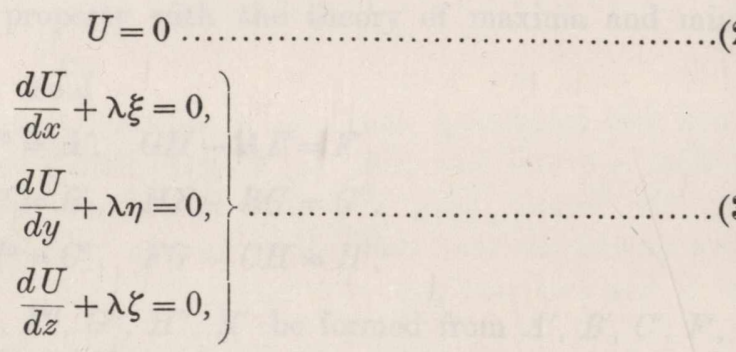

From the equations (2), (3), we obtain immediately

$$
\Theta=\xi x+\eta y+\zeta z=0
$$

and thence

$$
\Theta x=0, \quad \Theta y=0, \quad \Theta z=0
$$


so that a single equation more, such as

$$
\Phi=0 \text {. }
$$

where $\Phi$ is homogeneous and of the second order in $x, y, z$, would, in conjunction with the equations (3) and (5), enable us to eliminate linearly the seven quantities $x^{2}, y^{2}, z^{2}$, $y z, z x, x y, \lambda$. Such an equation may be thus obtained.

Let $L, M, N, R, S, T$, be the second differential coefficients of $U$, each of them divided by two. The equations (3) may be written

$$
\begin{aligned}
& L x+T y+S z+\lambda \xi=0 \\
& T x+M y+R z+\lambda \eta=0 \\
& S x+R y+N z+\lambda \xi=0 .
\end{aligned}
$$

And joining to these the equation (4),

$$
\xi x+\eta y+\zeta z \quad=0
$$

we have, by the elimination of $x, y, z$, in so far as they explicitly appear, and $\lambda$, an equation $\Phi=0$ of the required form. Hence we may write

$$
\Phi=-\left|\begin{array}{llll}
L, & T, & S, & \xi \\
T, & M, & R, & \eta \\
S, & R, & N, & \zeta \\
\xi, & \eta, & \zeta, &
\end{array}\right|
$$

or substituting for $L, M, N, R, S, T$, and expanding,

$$
\Phi=A x^{2}+B y^{2}+C z^{2}+2 F y z+2 G z x+2 H x y
$$

where the values of $A, B, C, F, G, H$ are

[I omit these values (10), and the values in the subsequent equations (13), (14), (20): the values (10). and (13) serve for the calculation of (14), $F U$, the expression for which with the letters $(a, b, c, f, g, h, i, j, k, l)$ in place of $\left(a, b, c, i, j, k, i_{1}, j_{1}, k_{1}, l\right)$ is reproduced in my "Third Memoir on Quantics," Phil. Trans. vol. CxLv.I. (1856) pp. 627-647: the values (20) serve for finding that of $(21), K(U)$, but the developed expression of this has not been calculated.]

Performing the elimination indicated, the result may be represented by

$$
\mathbf{F} U=\left|\begin{array}{ccccccc}
a, & k_{1}, & j, & l, & j_{1}, & k, & \xi \\
k, & b, & i_{1}, & i, & l, & k_{1}, & \eta \\
j_{1}, & i, & c, & i_{1}, & j, & l, & \zeta \\
2 \xi, & . & . & . & \zeta, & \eta & . \\
. & 2 \eta & . & \zeta & . & \xi & . \\
. & . & 2 \zeta & \eta & \xi & . & . \\
A & B & C & F & G & H & .
\end{array}\right|=0
$$

Partially expanding,

$$
\mathbf{F} U=A \mathrm{a}+B \mathrm{~b}+C \mathrm{c}+2 F \mathrm{f}+2 G \mathrm{~g}+2 H \mathrm{~h}
$$


The values of the coefficients $a, b, c, f, g, h$ may be useful on other occasions: they are as follows.

Substituting these values the result after all reductions becomes

$$
0=F(U)=
$$

It would be desirable, in conjunction with the above, to obtain the equation

$$
K(U)=0 \text {, }
$$

which results from the elimination of $x, y, z$ from the equations

$$
\frac{d U}{d x}=0, \quad \frac{d U}{d y}=0, \quad \frac{d U}{d z}=0
$$

(i.e. the condition of a curve of the third order having a multiple point), but to effect this would be exceedingly laborious. The following is the process of the elimination as given by Dr Hesse, Crelle, t. XxvirI. (and which applies also to the case of any three equations of the second order). Forming the function $\nabla U$, of the third order in $x, y, z$, by means of the equation

$$
\nabla U=\mid \begin{array}{ccc}
L, & T, & S \\
T, & M, & R \\
S, & R, & N
\end{array}
$$

( $L, M, N, R, S, T$, the same as before).

Then, in consequence of the equations (15), we have not only

$$
\nabla U=0 .
$$

which is very easily proved to be the case, but also

$$
\frac{d}{d x} \nabla U=0, \frac{d}{d y} \nabla U=0, \quad \frac{d}{d z} \nabla U=0
$$

as will be shown in a subsequent paper "On Points of Inflection." [I think never written.]

And from the six equations (15), (18), the six quantities $x^{2}, y^{2}, z^{2}, y z, z x, x y$, may be linearly eliminated: we have

$$
\nabla U=A x^{3}+B y^{3}+C z^{3}+3 I y^{2} z+3 J z^{2} x+3 K x^{2} y+3 I_{1} y z^{2}+3 J_{1} z x^{2}+3 K_{1} x y^{2}+6 \Lambda x y z \ldots(19),
$$

where the values of the coefficients $A, B, \ldots \Lambda$ are

and the result of the elimination is

$$
K(U)=\left|\begin{array}{llllll}
a, & k_{1}, & j, & l, & j_{1}, & k \\
k, & b, & i_{1}, & i, & l, & k_{1} \\
j_{1}, & i, & c, & i_{1}, & j, & l \\
A, & K_{1}, & J, & L, & J_{1}, & K \\
K, & B, & I_{1}, & I, & L, & K_{1} \\
J_{1}, & I, & C, & I_{1}, & J, & L
\end{array}\right|=0
$$

$\{K(U)$ is consequently, as is well known, a function of the twelfth order in $a, b, c$, $\left.i, j, k, i_{1}, j_{1}, k_{1}, l\right\}$. 
The equation

$$
\nabla U=0,
$$

combined with that of the curve, determine, as Dr Hesse has demonstrated in the paper quoted, the points of inflection of the curve. It may be inferred from this, that if $U$ reduce itself to the form

$$
U=\left(\alpha x^{2}+\beta y^{2}+\gamma z^{2}+2 \iota y z+2 \kappa x z+2 \lambda x y\right) P=V P .
$$

$P$ a linear function of $x, y, z$ : then $\nabla U$ takes the form

$$
\nabla U=P\left(\rho V+\sigma P^{2}\right)
$$

where $\rho$ is of the second order in the coefficients of $P$, and also in the coefficients $\alpha, \beta, \gamma, \iota, \kappa, \lambda$ : and $\sigma$ is equal to the determinant

$$
\left|\begin{array}{lll}
\alpha, & \lambda, & \kappa \\
\lambda, & \beta, & \iota \\
\kappa, & \iota, & \gamma
\end{array}\right|
$$

multiplied by a numerical factor. If $U$ is of the form

$$
U=P Q R \text {. }
$$

then

$$
\nabla U=\rho P Q R=\rho U
$$

and this equation is consequently the condition of the function $U$ being resolvable into linear factors. The equation in question resolves itself into

$$
\frac{A}{a}=\frac{B}{b}=\frac{C}{c}=\frac{I}{i}=\frac{J}{j}=\frac{K}{k}=\frac{I_{1}}{i_{1}}=\frac{J_{1}}{j_{1}}=\frac{K_{1}}{k_{1}}=\frac{\Lambda}{l}
$$

a system which must contain three independent equations only. It would be interesting to verify this $\grave{a}$ posteriori. 